\title{
$\mathrm{TiO}_{2} / \mathrm{SiO}_{2}$ 纳米薄膜的光催化活性和亲水性 *
}

\author{
余家国赵修建陈文梅林立张艾丽 \\ (武汉理工大学材料复合新技术国家重点实验室, 武汉 430070)
}

\begin{abstract}
摘要 通过 sol-gel 工艺在钠铝玻璃表面制备了均匀透明的 $\mathrm{TiO}_{2} / \mathrm{SiO}_{2}$ 复合纳米薄膜. 实验结果表明: 当 $\mathrm{SiO}_{2}$ 添 加量较高时, $\mathrm{TiO}_{2} / \mathrm{SiO}_{2}$ 复合纳米薄膜的光催化活性明显降低; 当 $\mathrm{SiO}_{2}$ 添加量较低时, $\mathrm{TiO}_{2} / \mathrm{SiO}_{2}$ 复合薄膜的光催 化活性无明显变化. 在 $\mathrm{TiO}_{2}$ 薄膜中添加 $\mathrm{SiO}_{2}$, 可以抑制薄膜中 $\mathrm{TiO}_{2}$ 晶粒的长大, 同时薄膜表面的羟基含量增加, 水在复合薄膜表面的润湿角下降, 亲水能力增强. 当 $\mathrm{SiO}_{2}$ 含量为 $10 \%-20 \%$ (摩尔分数) 时获得了润湿角为 0 的 超亲水性薄膜。
\end{abstract}

关键词： $\mathrm{TiO}_{2} / \mathrm{SiO}_{2}$ 复合纳米薄膜，溶胶 - 凝胶工艺，光催化活性，超亲水性

近年来, 已有许多关于将 $\mathrm{TiO}_{2}$ 光催化剂固定 于玻璃、陶瓷面砖和卫生洁具等物品表面, 从而赋予 它们具有杀菌和自洁净等功能的研究 ${ }^{[1-4]}$. 已有研 究表明 ${ }^{[4]}$ : 超亲水性对于 $\mathrm{TiO}_{2}$ 光催化薄膜表面的自 洁净、易清洗等性能具有十分重要的影响, 因而有 关这方面的研究工作越来越多. Fujishima 等 ${ }^{[5]}$ 发现 $\mathrm{TiO}_{2}$ 薄膜在紫外光照射下具有超亲水性. 实际应 用要求水在 $\mathrm{TiO}_{2}$ 薄膜表面的润湿角在光照停止时, 上升比较缓慢或始终维持在 $0^{\circ}$ 附近. 为了改善 $\mathrm{TiO}_{2}$ 薄膜表面的亲水性, 我们通过改变 $\mathrm{TiO}_{2}$ 薄膜 表面的微结构如表面粗糙度和表面差基含量, 可明 显改善薄膜自身的亲水特性 ${ }^{[6]}$. 最新研究 ${ }^{[7]}$ 发现: 由 $\mathrm{TiO}_{2}$ 和 $\mathrm{SiO}_{2}$ 混合溶胶在陶瓷面砖表面制备的复合 薄膜在光诱导下具有超亲水性, 且超亲水性维持也 较好. 但有关以醇盐为原料, 采用溶胶 - 凝胶工艺 在钠钙玻璃表面制备 $\mathrm{TiO}_{2} / \mathrm{SiO}_{2}$ 超亲水光催化透明 复合薄膜的研究的文献报导不多. 为此, 本文研究 了 $\mathrm{SiO}_{2}$ 添加量对于 $\mathrm{TiO}_{2} / \mathrm{SiO}_{2}$ 超亲水光催化复合 纳米薄膜的透光率、亲水性和光催化活性的影响.
用正硅酸乙脂 (TEOS) 和钛酸丁脂作为合成前 驱体溶液的原料, 采用文献 [8] 报导的方法制备 $\mathrm{TiO}_{2} / \mathrm{SiO}_{2}$ 复合 薄膜, 复合薄膜中 $\mathrm{SiO}_{2}$ 的含量 $(x$, 摩尔分数,下同)分别为 $0 、 5 \% 、 10 \% 、 15 \% 、 20 \%$ 、 $33 \%$ 和 $50 \%$.

\section{2 物性分析测试}

在 KRATOS XSAM 800 型电子能谱仪上对 $\mathrm{TiO}_{2} / \mathrm{SiO}_{2}$ 复合薄膜进行了 XPS 研究. $X$ 射线源为 $\mathrm{Mg} K_{\alpha}$ 射线 (1 $253.6 \mathrm{eV}$ ), 实验过程中分析室的真空 度优于 $2 \times 10^{-7} \mathrm{~Pa}$, 电流电压为 $16 \mathrm{~mA} \times 12.5 \mathrm{kV}$. 分析器模式: FRR. 以样品表面来自 XPS 仪器本身 的油污染碳 $\left(\mathrm{C} 1 s, E_{\mathrm{b}}=284.80 \mathrm{eV}\right)$ 作为荷电校正标 准. 用 $60 \mathrm{SXB}$ 型富里叶变换红外光谱仪 (FTIR) 研 究了 $\mathrm{TiO}_{2} / \mathrm{SiO}_{2}$ 凝胶的物相变化过程. 用 UV-160A 型紫外可见光光度计测量了 $\mathrm{TiO}_{2} / \mathrm{SiO}_{2}$ 薄膜在 200-800 nm 波长范围内的透过率. $\mathrm{TiO}_{2} /$ $\mathrm{SiO}_{2}$ 复合薄膜表面的亲水性评价按文献 [8]报导的 方法进行. 薄膜的光催化活性评价按文献 [9]报导 的方法进行.

\section{1 实验部分}

\section{$1.1 \mathrm{TiO}_{2} / \mathrm{SiO}_{2}$ 复合纳米薄膜的制备}

\section{2 实验结果与讨论}

\section{$2.1 \mathrm{TiO}_{2} / \mathrm{SiO}_{2}$ 复合纳米薄膜的透光率}




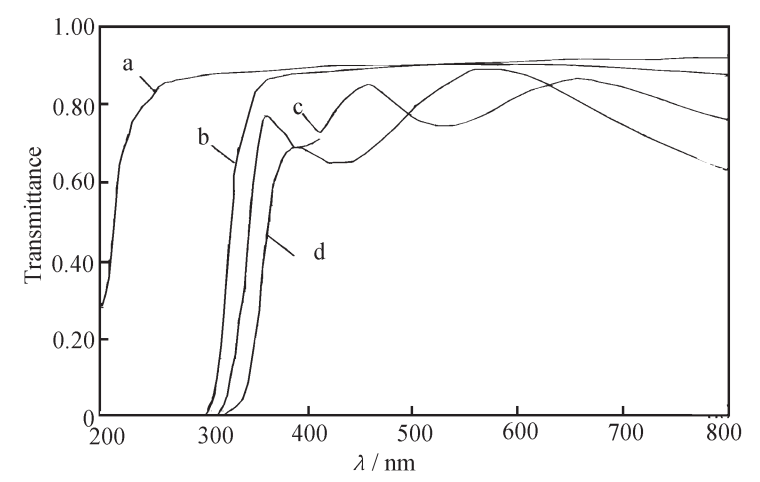

图 1 几种镀膜玻璃和石英玻璃的紫外可见光透光率曲线

Fig. 1 UV-VIS spectra of fused quartz and soda lime glass

(a) fused quartz, soda lime glass coated (b) $\mathrm{SiO}_{2}$ film,

(c) $\mathrm{TiO}_{2} / \mathrm{SiO}_{2}$ films and (d) $\mathrm{TiO}_{2}$ films

图 1 示出几种镀膜玻璃和石英玻璃的紫外可见 光透光率曲线. 从图 1 可以看出, 石英玻璃的透光 率最高, 而且在近紫外波段几乎没有吸收. 这是由 于石英玻璃是绝缘体, 它的价带和导带之间的禁带 宽度很大 $(11.7 \mathrm{eV})^{[10]}$, 近紫外光不能使电子从价 带向导带跃迁, 因此石英玻璃在近紫外波段几乎没 有吸收. 从曲线 $\mathrm{b}$ 可以看出, 镀 $\mathrm{SiO}_{2}$ 膜的普通玻 璃的吸收边缘波长在 $300 \mathrm{~nm}$ 附近. 这是由于普通 玻璃的本征吸收引起的, 因为 $\mathrm{SiO}_{2}$ 膜在波长 300 $\mathrm{nm}$ 附近没有吸收. 曲线 $\mathrm{d}$ 与 $\mathrm{b}$ 比较, 镀 $\mathrm{TiO}_{2}$ 膜的 普通玻璃的吸收边缘波长发生了明显的红移, 其吸 收边缘波长在 $350 \mathrm{~nm}$ 附近. $\mathrm{TiO}_{2}$ 的禁带宽度为 $3.2 \mathrm{eV}$, 其吸收边缘波长应为 $387.5 \mathrm{~nm}$. 然而, 溶 胶 - 凝胶工艺制备的 $\mathrm{TiO}_{2}$ 膜晶粒尺寸较小, 由于量 子尺寸效应, $\mathrm{TiO}_{2}$ 的禁带变宽, 因而吸收边缘波长 显示了“蓝移” [9]. 曲线 $\mathrm{c}$ 与曲线 $\mathrm{d}$ 比较, 虽然两者 的镀膜次数和热处理时间相同, 但镀 $\mathrm{TiO}_{2} / \mathrm{SiO}_{2}$ 复 合膜的普通玻璃的吸收边缘波长却发生了明显的蓝 移. 这是由于 $\mathrm{TiO}_{2} / \mathrm{SiO}_{2}$ 复合膜中 $\mathrm{TiO}_{2}$ 的晶粒尺寸 较小引起的. 这说明, 在 $\mathrm{TiO}_{2}$ 膜中添加 $\mathrm{SiO}_{2}$ 抑制 了 $\mathrm{TiO}_{2}$ 的晶粒长大 ${ }^{[8]}$.

\section{$2.2 \mathrm{TiO}_{2} / \mathrm{SiO}_{2}$ 凝胶的红外光谱研究}

图 2 为 $\mathrm{TiO}_{2} / \mathrm{SiO}_{2}(1: 1)$ 凝胶在不同温度下保 温 $1 \mathrm{~h}$ 热处理后 (在空气中放置 2 天) 的红外光谱 图. 图中 $3000 \sim 3800 \mathrm{~cm}^{-1}$ 区域的吸收峰是 $-\mathrm{OH}$ 基团伸缩振动引起的, 是各种 $-\mathrm{OH}$ (如 $\mathrm{Si}-\mathrm{O}-$ $\mathrm{OH} 、 \mathrm{Ti}-\mathrm{O}-\mathrm{OH}$ ) 峰的叠加, 与结构水有关. 2860 $2950 \mathrm{~cm}^{-1}$ 的峰为亚甲基弯曲振动所产生的. 1637

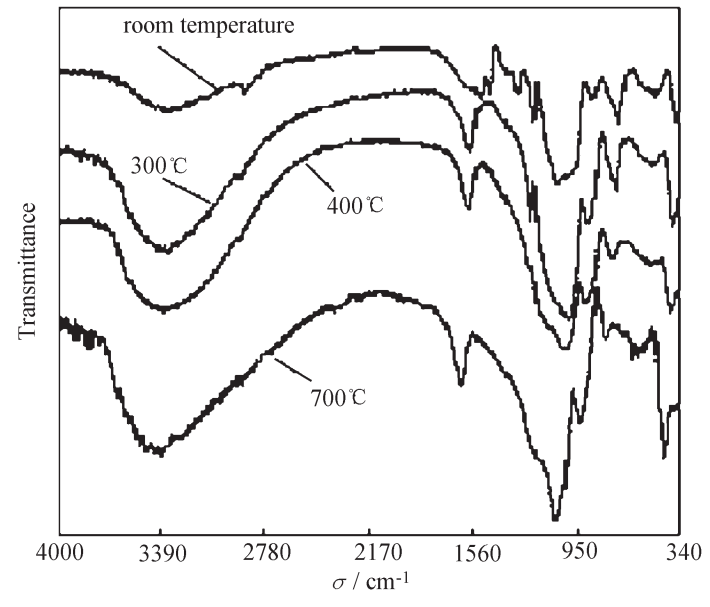

图 $2 \mathrm{TiO}_{2} / \mathrm{SiO}_{2}(1: 1)$ 凝胶在不同温度下保温 $1 \mathrm{~h}$ 热处理后的红外光谱图

Fig. 2 Infrared spectra of $\mathrm{TiO}_{2} / \mathrm{SiO}_{2}(1: 1)$ gels heat-treated at different temperatures for $\mathbf{1 ~} \mathrm{h}$ and then placed in air for $\mathbf{2}$ days

$\mathrm{cm}^{-1}$ 处的吸收峰是物理吸附水的 $\mathrm{O}-\mathrm{H}$ 键的弯曲 振动. $1532 \mathrm{~cm}^{-1} 、 1590 \mathrm{~cm}^{-1}$ 和 $1367 \mathrm{~cm}^{-1}$ 的吸收峰 可归因于 $\mathrm{CH}_{3}$ 和 $\mathrm{CH}_{2}$ 基团中 $\mathrm{C}-\mathrm{H}$ 链的弯曲振动 所引起的. 位于 $1000 \sim 1250 \mathrm{~cm}^{-1}$ 范围内不对称的 肩峰是由 $\mathrm{Si}-\mathrm{O}-\mathrm{Si}$ 键非对称的伸缩振动引起的; 对称性振动 $775 \mathrm{~cm}^{-1}$ 和 $440 \mathrm{~cm}^{-1}$ 的峰分别来自于 $\mathrm{Si}-\mathrm{O}-\mathrm{Si}$ 键和 $\mathrm{Ti}-\mathrm{O}-\mathrm{Ti}$ 键的贡献. 而在 935 $\mathrm{cm}^{-1}$ 的峰则是 $\mathrm{Si}-\mathrm{O}-\mathrm{Ti}$ 和 $\mathrm{Si}-\mathrm{OH}$ 引起的. 由图 2 可以看出, $\mathrm{TiO}_{2} / \mathrm{SiO}_{2}(1: 1)$ 凝胶经热处理后, 有机 基团的振动峰逐渐减弱. 至 $400{ }^{\circ} \mathrm{C}$, 有机基团完全 消失.从图 2 还可以看出, 随着热处理温度逐渐升 高到 $700{ }^{\circ} \mathrm{C}, \mathrm{TiO}_{2} / \mathrm{SiO}_{2}$ (1: 1) 凝胶中的表面吸附水 和化学结合水不但没有消失, 反而有增大的趋势. 其原因可能是 $\mathrm{SiO}_{2}$ 或 $\mathrm{TiO}_{2}$ 分子网络中的有机基团 逐渐被炭化分解. $\mathrm{SiO}_{2}$ 具有很强的亲水和吸附水的 能力, 当这些样品放置在空气中, 吸附了大量的水, 并有部分水转变为化学结合水.

\section{$2.3 \mathrm{TiO}_{2} / \mathrm{SiO}_{2}(1: 1)$ 薄膜表面的羟基含量}

图 3 给出 $\mathrm{TiO}_{2} / \mathrm{SiO}_{2}(1: 1)$ 复合薄膜表面 $\mathrm{O} l \mathrm{~s}$ 的高分辨 XPS 谱, 其拟合结果列于表 1 中. 从图 3 和表 1 可以看出, O $1 s$ 的高分辨 XPS 谱可以分解 成 4 个小峰, 分别来自 (a) $\mathrm{Ti}-\mathrm{O}$ 键、 (b) $\mathrm{Si}-\mathrm{O}$ 键、 (c) $-\mathrm{OH}$ 基团和 (d) $\mathrm{C}-\mathrm{O}$ 键. 各种氧所占的百分 率分别为 $21.3 \% 、 36.0 \% 、 26.9 \%$ 和 $15.8 \%$. 结合 前述的红外光谱结果可知, 所制备的 $\mathrm{TiO}_{2} / \mathrm{SiO}_{2}(1$ : 


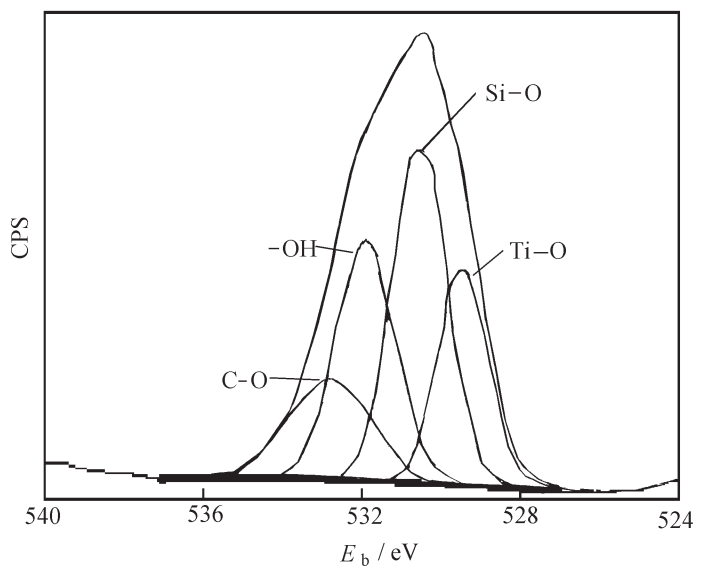

图 $3 \mathrm{TiO}_{2} / \mathrm{SiO}_{2}(1: 1)$ 复合薄膜表面 $\mathrm{O} 1 s$ 的高分辨 XPS 谱

Fig. 3 XPS spectrum of the $01 s$ region on the surface of a nano- $\mathrm{TiO}_{2} / \mathrm{SiO}_{2}(1: 1)$ film

1)复合薄膜表面含有大量羟基。其表面羟基含量高 于普通 $\mathrm{TiO}_{2}$ 薄膜表面的差基含量 ${ }^{[11]}$.

\section{$2.4 \mathrm{TiO}_{2} / \mathrm{SiO}_{2}$ 复合薄膜的光催化活性和亲水性}

从表 2 可以看出: 随着在 $\mathrm{TiO}_{2}$ 薄膜中 $\mathrm{SiO}_{2}$ 添 加量的增加, $\mathrm{TiO}_{2}$ 薄膜的光催化活性下降; 但当 $\mathrm{SiO}_{2}$ 添加量 (摩尔分数, $x$ ) 小于 $20 \%$ 时, $\mathrm{TiO}_{2}$ 薄膜 的光催化活性下降不明显; 当 $\mathrm{SiO}_{2}$ 添加量大于 $33 \%$ 时, $\mathrm{TiO}_{2}$ 薄膜的光催化活性下降明显. 这可能 是由于下述原因引起的.一方面, 在 $\mathrm{TiO}_{2}$ 薄膜中 少量添加 $\mathrm{SiO}_{2}$, 可以抑制薄膜中 $\mathrm{TiO}_{2}$ 的晶粒长 $大^{[8,12]}$, 使 $\mathrm{TiO}_{2}$ 的禁带宽度增加, $\mathrm{TiO}_{2}$ 的氧化还原 能力增强; $\mathrm{TiO}_{2}$ 薄膜的光催化活性增强; 同时, $\mathrm{TiO}_{2}$ 薄膜表面的差基含量增加, 也可以提高薄膜的光催 化活性 ${ }^{[13]}$, 由于 $\mathrm{SiO}_{2}$ 的量较少, 不会影响 $\mathrm{TiO}_{2}$ 薄 膜中锐钛矿相的形成, 所以 $\mathrm{TiO}_{2}$ 薄膜中虽然少量
表 $1 \mathrm{TiO}_{2} / \mathrm{SiO}_{2}(1: 1)$ 复合薄膜表面 $\mathrm{O} 1 s$ 的高分辨 XPS 谱的拟合结果

Table 1 Peak fitting results of the spectra of the O $1 s$ region on the surface of a nano- $\mathrm{TiO}_{2} / \mathrm{SiO}_{2}(1: 1)$ film

\begin{tabular}{|c|c|c|c|c|}
\hline & $\mathrm{O} 1 s(\mathrm{Ti}-\mathrm{O})$ & $\mathrm{O} 1 s(\mathrm{Si}-\mathrm{O})$ & $\mathrm{O} 1 s(-\mathrm{OH})$ & O $1 s(\mathrm{C}-\mathrm{O})$ \\
\hline$E_{\mathrm{b}} / \mathrm{eV}$ & 529.45 & 530.55 & 531.90 & 532.80 \\
\hline$r_{\mathrm{i}}$ & 0.213 & 0.360 & 0.269 & 0. 158 \\
\hline
\end{tabular}

$r_{\text {i }}$ represents the ratio $A_{i} / \Sigma A_{i}\left(A_{i}\right.$ is the area of each peak ).

添加了无光催化活性的 $\mathrm{SiO}_{2}$, 薄膜的光催化活性下 降不明显. 另一方面, 当 $\mathrm{SiO}_{2}$ 添加量较大时, 虽然 可以增加 $\mathrm{TiO}_{2}$ 薄膜表面的羟基含量和抑制晶粒长 大, 但 $\mathrm{SiO}_{2}$ 可以在薄膜中形成 $\mathrm{Si}-\mathrm{O}$ 网络, 抑制锐 钛矿相的形成 ${ }^{[12]}$, 同时薄膜表面无光催化活性的 $\mathrm{SiO}_{2}$ 所占的比例较大, 因而 $\mathrm{TiO}_{2}$ 薄膜的光催化活 性下降明显。

从表 2 还可以看出, 随着 $\mathrm{TiO}_{2}$ 薄膜中 $\mathrm{SiO}_{2}$ 添 加量的增加, 水在 $\mathrm{TiO}_{2}$ 薄膜表面的润湿角下降. 当加人量为 $10 \%-20 \%$ 时, 水在 $\mathrm{TiO}_{2}$ 薄膜表面的 润湿角下降为 $0^{\circ}$; 但当加人量高于 $33 \%$ 时, 水在 $\mathrm{TiO}_{2}$ 薄膜表面的润湿角有回升的趋势, 其原因尚不 清楚, 目前正在研究中. $\mathrm{TiO}_{2} / \mathrm{SiO}_{2}$ 复合薄膜表面 出现超亲水性的主要原因是由于一方面薄膜表面羟 基增加、极性增强; 另一方面, $\mathrm{TiO}_{2}$ 的晶粒变小, 薄 膜比表面积增大, 吸附能力增强引起的.

\section{3 结 论}

(1) 在 $\mathrm{TiO}_{2}$ 薄膜中添加 $\mathrm{SiO}_{2}$, 可以抑制 $\mathrm{TiO}_{2} /$ $\mathrm{SiO}_{2}$ 复合薄膜中 $\mathrm{TiO}_{2}$ 晶粒的长大.

(2) 在 $\mathrm{TiO}_{2}$ 薄膜中添加 $\mathrm{SiO}_{2}$, 薄膜表面的羟基 含量增加, 水在复合薄膜表面的润湿角下降, 复合

表 $2 \mathrm{SiO}_{2}$ 添加量对 $\mathrm{TiO}_{2}$ 薄膜的光催化活性和亲水性的影响

Table 2 Effect of $\mathrm{SiO}_{2}$ addition on phoptocatalytic activity and hydrophilic property of $\mathrm{TiO}_{2}$ films

\begin{tabular}{lccccccc}
\hline $\mathrm{SiO}_{2}$ content $(x)$ & 0 & 0.05 & 0.10 & 0.15 & 0.20 & 0.33 & 0.50 \\
\hline$k / \mathrm{min}^{-1}$ & 0.0189 & 0.0191 & 0.0185 & 0.0178 & 0.0173 & 0.0153 & 0.0137 \\
$t_{1 / 2} /$ min & 36.7 & 36.3 & 37.5 & 38.9 & 40.1 & 45.3 & 50.6 \\
\hline Contact angle $\theta /\left(^{\circ}\right)$ & 15 & 5 & 0 & 0 & 0 & 5 & 10 \\
\hline
\end{tabular}

Film thickness $0.18 \mu \mathrm{m}$ or so ( 1 coating cycle), $x$ : mole fraction. 
薄膜表面亲水能力增强 .

(3) 当 $\mathrm{TiO}_{2}$ 薄膜中 $\mathrm{SiO}_{2}$ 的添加量较小时, $\mathrm{TiO}_{2}$ 薄膜的光催化活性下降不明显, 当 $\mathrm{SiO}_{2}$ 添加量较大 时, $\mathrm{TiO}_{2}$ 薄膜的光催化活性下降明显.

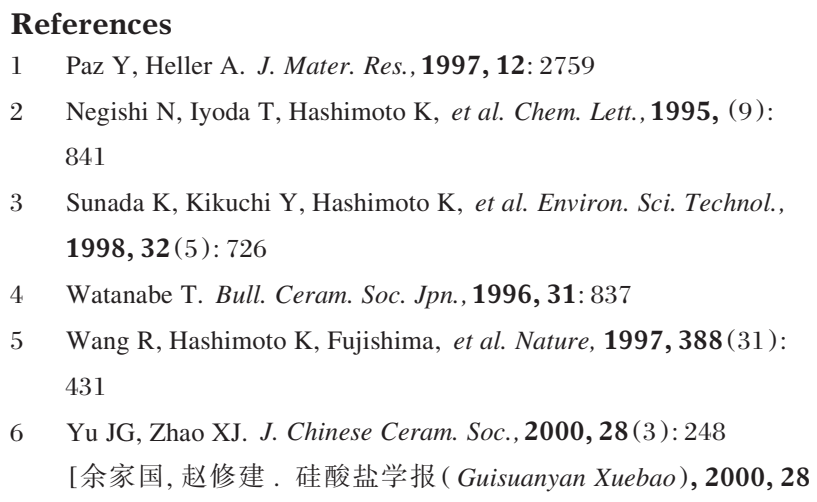

6 Yu JG, Zhao XJ. J. Chinese Ceram. Soc., 2000, 28 (3): 248

[余家国, 赵修建。硅酸盐学报 (Guisuanyan Xuebao), 2000, 28

(3): 248 ]

7 Machida M, Norimoto K, Watanabe T, et al. J. Mater. Sci., 1999, 34: 2569

8 Yu JG, Zhao XJ, Lin L, et al. J. Inorg. Mater., Accepted, to be published[余家国, 赵修建, 林 立等. 无机材料学报 (Wuji Cailiao Xuebao), 接受, 待发表]

9 Yu Jiaguo, Zhao Xiujian, Zhao Qingnan. J. Mater. Sci. Lett., 2000, 19(12): 1015

10 Gan FX. Science and Technology of Modern Glass (First volume). Shanghai: Shanghai Sci. Tech. Press, 1988. 322 [干福喜。现代玻 璃科学技术 (上), 上海: 上海科学技术出版社, 1988.322]

11 Yu JG, Zhao XJ. Chinese J. Catal., 2000, 21 (3): 213 [余家国, 赵 修建．催化学报 (Cuihua Xuebao), 2000, 21(3): 213]

12 Lin Jun, Yu Jimmy C, Lo D, et al. J. Catal., 1999, 183: 368

13 Yu Jiaguo, Zhao Xiujian, Du Jincheng, et al. J. Sol-Gel Sci. Tech., 2000, 17: 163

\title{
Photocatalytic Activity and Hydrophilic Property of $\mathrm{TiO}_{2} / \mathrm{SiO}_{2}$ Composite Nanometer Thin Films *
}

\author{
Yu Jia-Guo Zhao Xiu-Jian Chen Wen-Mei Lin Li Zhang Ai-Li \\ ( State Key Laboratory of Advanced Technology for Materials Synthesis and Processing Wuhan University of Technology, Wuhan \\ 430070)
}

\begin{abstract}
The uniform transparent $\mathrm{TiO}_{2} / \mathrm{SiO}_{2}$ composite nanometer thin films were prepared via sol-gel processing on the soda lime glass substrates. The photocatalytic activity of the as-prepared $\mathrm{TiO}_{2} / \mathrm{SiO}_{2}$ composite nanometer thin films obviously decreases when the amount of $\mathrm{SiO}_{2}$ addition is large. However, the photocatalytic activity has no obvious change when the amount of $\mathrm{SiO}_{2}$ addition is small (Table 2). The addition of $\mathrm{SiO}_{2}$ can suppress the grain growth of $\mathrm{TiO}_{2}$ crystal, increase the hydroxyl content of $\mathrm{TiO}_{2}$ film, decrease the contact angle for water of $\mathrm{TiO}_{2}$ films and enhance the hydrophilic property of $\mathrm{TiO}_{2}$ films. The super-hydrophilic $\mathrm{TiO}_{2} / \mathrm{SiO}_{2}$ composite nanometer films with the contact angle of $0^{\circ}$ were obtained by the addition of $10 \%-20 \%$ (mole fraction) $\mathrm{SiO}_{2}$.
\end{abstract}

Keywords: $\quad \mathrm{TiO}_{2} / \mathrm{SiO}_{2}$ composite nanometer thin films, Sol-gel processing, Photocatalytic activity, Super-hydrophilic property 